\title{
CLASSIFICATION OF QUATERNIONIC HYPERBOLIC ISOMETRIES
}

\author{
KRISHNENDU GONGOPADHYAY AND SHIV PARSAD
}

\begin{abstract}
Let $\mathbb{F}$ denote either the complex numbers $\mathbb{C}$ or the quaternions $\mathbb{H}$. Let $\mathbf{H}_{\mathbb{F}}^{n}$ denote the $n$-dimensional hyperbolic space over $\mathbb{F}$. We obtain algebraic criteria to classify the isometries of $\mathbf{H}_{\mathbb{F}}^{n}$. This generalizes the work in Geom. Dedicata 157 (2012), 23-39 and Proc. Amer. Math. Soc. 141 (2013), 10171027 , to isometries of arbitrary dimensional quaternionic hyperbolic space. As a corollary, a characterization of isometries of $\mathbf{H}_{\mathbb{C}}^{n}$ is also obtained.
\end{abstract}

\section{INTRODUCTION}

Classically, the isometries of the real hyperbolic space $\mathbf{H}_{\mathbb{R}}^{n}$ are classified as elliptic, parabolic and hyperbolic according to the dynamics of their fixed points. In two and three dimensional real hyperbolic geometries, this trichotomy of the isometries are classified algebraically in terms of their traces; cf. 3. Theorems 4.3.1 and 4.3.4]. There have been several attempts to generalize this algebraic classification in higher dimensional real hyperbolic geometry; for example, see [1,23, 24 for an approach using Clifford numbers, and [7,12, for algebraic classification in four and five dimensional real hyperbolic geometries using quaternionic Möbius transformations. Similar trichotomy based on the fixed-point dynamics of the isometries is also valid in the complex and the quaternionic hyperbolic geometries. In order to understand the geometry and dynamics of isometries in these geometries, it is a natural question to ask for similar criteria to classify the isometries using conjugacy invariants (like trace). The isometry group of $\mathbf{H}_{\mathbb{C}}^{1}$ and $\mathbf{H}_{\mathbb{H}}^{1}$ can be identified with the orientation-preserving isometry group of $\mathbf{H}_{\mathbb{R}}^{2}$ and $\mathbf{H}_{\mathbb{R}}^{4}$ respectively and hence, using our knowledge of real hyperbolic geometry, the isometries can be classified algebraically. In two dimensional complex hyperbolic geometry, similar classification is known due to Goldman [11, Theorem 6.2.4]; see also Giraud [10.

The quaternionic hyperbolic geometry is not so widely studied unlike its real and complex counterparts. In analogy with the complex hyperbolic geometry, a systematic study of the quaternionic hyperbolic geometry using the Siegel domain model was initiated by Kim and Parker [17] relatively recently; see also [2]. Many of the results in real and complex hyperbolic geometries naturally carry over to the quaternionic hyperbolic case; cf. [8]. However, there are many aspects of the complex hyperbolic geometry that do not carry over naturally to the quaternionic hyperbolic setting; cf. [6, 17]. One obstruction in such a generalization from the

Received by the editors August 1, 2012.

2010 Mathematics Subject Classification. Primary 51M10; Secondary 15B33, 15B57, 20 G20.

Key words and phrases. Complex and quaternionic hyperbolic space, classification of isometries.

The first author acknowledges the support of SERC-DST FAST grant SR/FTP/MS-004/2010.

The second author acknowledges the support of CSIR research fellowship. 
complex to the quaternions is the noncommutativity of the quaternions. In particular, algebraic criteria to classify the isometries of the quaternionic hyperbolic space is also not straightforward. This is because the conjugacy invariants like trace and the characteristic polynomial, used in the real and complex hyperbolic cases, no longer served as conjugacy invariants for matrices over the quaternions; cf. [26].

In this paper our aim is to obtain algebraic criteria to classify isometries of the $n$ dimensional quaternionic hyperbolic space. In [5], Cao and the first-named author have offered a counterpart of Goldman's theorem in the two dimensional quaternionic hyperbolic geometry. Recently, algebraic characterization of isometries of $\mathbf{H}_{\mathbb{H}}^{3}$ has been obtained by the first-named author in [13. The key idea used in [5, 13. involves an embedding of the quaternions into the matrix ring $M_{2}(\mathbb{C})$ and classical analysis of the nature of roots of a real cubic or biquadratic equation. Generalizing this approach to arbitrary dimension, we have obtained algebraic criteria to classify isometries of the $n$-dimensional quaternionic hyperbolic space $\mathbf{H}_{\mathbb{H}}^{n}$ for arbitrary $n$; cf. Theorem 3.1. As a corollary, a characterization of isometries of $\mathbf{H}_{\mathbb{C}}^{n}$ is also obtained; cf. Corollary 3.2 For another classification of isometries of $\mathbf{H}_{\mathbb{C}}^{n}$, which is closer to the spirit of Goldman's theorem, see [15].

In section 2 we review the basic notions and tools needed for deriving the desired classification. We also refined the above trichotomy in this section; cf. section 2.4 . In section 3 , we state and prove our main result, Theorem 3.1

\section{Preliminaries}

2.1. The quaternions. Let $\mathbb{H}$ be the division ring of quaternions and $\mathbb{H}^{*}=\mathbb{H}-$ $\{0\}$. For a quaternion, resp. complex number $z$, let $\Re(z)$ denote the real part of $z$. Let $\Im(\mathbb{H})$ denote the purely imaginary subspace of $\mathbb{H}$. Let $V$ be a right vector space over $\mathbb{H}$ and let $T$ be an invertible linear transformation of $V$. For $v \in V$, $\lambda \in \mathbb{H}^{*}$, suppose $T v=v \lambda$, i.e., $\lambda$ is a (right) eigenvalue of $T$. For $\mu \in \mathbb{H}^{*}$, $T(v \mu)=(v \mu) \mu^{-1} \lambda \mu$, i.e., the eigenvalues of $T$ occur in similarity classes and if $v$ is a $\lambda$-eigenvector, then $v \mu \in v \mathbb{H}$ is a $\mu^{-1} \lambda \mu$-eigenvector. The one-dimensional right subspace of $V$ spanned by $v$ will be called $\lambda$-eigenline. Thus the eigenvalues are no more conjugacy invariants for $T$, but the similarity classes of eigenvalues are conjugacy invariant. Note that each similarity class of eigenvalues contains a unique pair of complex conjugate numbers. We may choose one of these complex eigenvalues to be the representative of its similarity class. In the following, we will follow the convention of identifying the similarity class of an eigenvalue with its complex representative $r e^{i \theta}, 0 \leq \theta \leq \pi$. For simplicity, often a similarity class of eigenvalue will be referred to as an 'eigenvalue'. Two eigenvalues are distinct if their similarity classes are mutually disjoint. For $A$ an $n \times n$ matrix, a 'minimal polynomial' of $A$ is the least degree monic polynomial satisfied by $A$. It follows from [26, Theorem 6.3] that every $n \times n$ quaternionic matrix is conjugate to an $n \times n$ complex matrix $A_{c}$. The algebraic multiplicity of an eigenvalue $r e^{i \theta}$ of $A_{c}$ is defined to be a multiplicity of the similarity class $\left[r e^{i \theta}\right]$ of eigenvalues of $A$.

For more properties of matrices over quaternions, cf. [26].

2.2. The space $\mathbf{H}_{\mathbb{F}}^{n}$. Let $\mathbb{F}$ denote either the field of complex numbers $\mathbb{C}$ or the division ring of the real quaternions $\mathbb{H}$. Consider the (right) vector space $\mathbb{V}=\mathbb{F}^{n+1}$ over $\mathbb{F}$ equipped with the Hermitian form of signature $(n, 1)$,

$$
\langle z, w\rangle=-\bar{z}_{0} w_{0}+\bar{z}_{1} w_{1}+\cdots+\bar{z}_{n} w_{n},
$$


where $z$ and $w$ are the column vectors in $\mathbb{V}$ with entries $z_{0}, \cdots, z_{n}$ and $w_{0}, \cdots, w_{n}$ respectively. The isometry group $U(n, 1 ; \mathbb{F})$ of $(\mathbb{V},\langle\rangle$,$) is denoted by \mathrm{U}(n, 1)$, resp. $\operatorname{Sp}(n, 1)$ for $\mathbb{F}=\mathbb{C}$, resp. $\mathbb{H}$. Define

$$
\mathbb{V}_{0}=\{z \in \mathbb{V} \mid\langle z, z\rangle=0\}, \mathbb{V}_{+}=\{z \in \mathbb{V} \mid\langle z, z\rangle>0\}, \mathbb{V}_{-}=\{z \in \mathbb{V} \mid\langle z, z\rangle<0\}
$$

A vector $v \in \mathbb{V}$ is called time-like, resp. space-like, resp. light-like if $v$ belongs to $\mathbb{V}_{-}$, resp. $\mathbb{V}_{+}$, resp. $\mathbb{V}_{0}$. A right subspace $\mathbb{W}$ of $\mathbb{V}$ is time-like if it is non-degenerate and contains a time-like vector. It is space-like, resp. light-like if $\left.\langle\rangle\right|_{,\mathbb{W}}$ is positivedefinite, resp. degenerate. If $\mathbb{W}$ is time-like, then the orthogonal complement $\mathbb{W}^{\perp}$ is space-like and vice-versa. Let $\left\{e_{0}, e_{1}, \cdots, e_{n}\right\}$ be the standard orthonormal basis of $\mathbb{V}$. Let $e_{0}^{\perp}$ be the orthogonal complement of $e_{0} \mathbb{F}$. We identify $e_{0}^{\perp}$ with $\mathbb{F}^{n} \subset \mathbb{F}^{n, 1}$. For $v, w \in \mathbb{F}^{n},\langle v, w\rangle=\bar{z}_{1} w_{1}+\cdots+\bar{z}_{n} w_{n}$. The isometry group of $\left(\mathbb{H}^{n},\langle\rangle,\right)$ is denoted by $\mathrm{U}(n ; \mathbb{F})$. Note that $\mathrm{U}(n ; \mathbb{F})=\mathrm{U}(n)$, resp. $\operatorname{Sp}(n)$ for $\mathbb{F}=\mathbb{C}$, resp. $\mathbb{H}$ and $\mathrm{U}(1 ; \mathbb{F})=\{\lambda I|| \lambda \mid=1\}$.

Let $\mathbb{P}(\mathbb{V})$ be the projective space obtained from $\mathbb{V}$, i.e., $\mathbb{P}(\mathbb{V})=\mathbb{V}-\{0\} / \sim$, where $u \sim v$ if there exists $\lambda$ in $\mathbb{F}^{*}$ such that $u=v \lambda$, and $\mathbb{P}(\mathbb{V})$ is equipped with the quotient topology. Suppose $\pi: \mathbb{V}-\{0\} \rightarrow \mathbb{P}(\mathbb{V})$ denotes the projection map. The $n$-dimensional hyperbolic space over $\mathbb{F}$ is defined to be $\mathbf{H}_{\mathbb{F}}^{n}=\pi\left(\mathbb{V}_{-}\right)$. The boundary $\partial \mathbf{H}_{\mathbb{F}}^{n}$ is $\pi\left(\mathbb{V}_{0}\right)$. The group $\mathrm{U}(n, 1 ; \mathbb{F})$ acts as the isometry group of $\mathbf{H}_{\mathbb{F}}^{n}$. The actual group of isometries of $\mathbf{H}_{\mathbb{F}}^{n}$ is $\mathrm{PU}(n, 1 ; \mathbb{F})=\mathrm{U}(n, 1 ; \mathbb{F}) / Z(\mathrm{U}(n, 1 ; \mathbb{F}))$, where $Z(\mathrm{U}(n, 1 ; \mathbb{F}))$ denotes the center. When $\mathbb{F}=\mathbb{C}, Z(\mathrm{U}(n, 1))$ is the circle group $\mathbb{S}^{1}=\{\lambda I|| \lambda \mid=1\}$, and for $\mathbb{F}=\mathbb{H}, Z(\operatorname{Sp}(n, 1))=\{I,-I\}$; here $I$ denotes the identity transformation in $\mathrm{U}(n, 1 ; \mathbb{F})$. Thus an isometry $g$ of $\mathbf{H}_{\mathbb{F}}^{n}$ lifts to a transformation $\tilde{g}$ in $\mathrm{U}(n, 1 ; \mathbb{F})$. However, for convenience, we mostly deal with the linear group $\mathrm{U}(n, 1 ; \mathbb{F})$ rather than $\mathrm{PU}(n, 1 ; \mathbb{F})$. Accordingly, we shall regard $\mathrm{U}(n, 1 ; \mathbb{F})$ as acting on $\mathbf{H}_{\mathbb{F}}^{n}$ as well as on $\mathbb{F}^{n, 1}$.

For more details on several models of $\mathbf{H}_{\mathbb{F}}^{n}$, see [8, 17, 20].

2.3. Discriminant system for real polynomials. We summarize 16, Theorem 1] below. This will be used to derive our criteria to classify isometries of $\mathbf{H}_{\mathbb{H}}^{n}$.

Given a polynomial $f(x)=a_{0} x^{n}+a_{1} x^{n-1}+\cdots+a_{n}$, write the first derivative of $f(x)$ as

$$
f^{\prime}(x)=0 . x^{n}+n a_{0} x^{n-1}+\cdots+a_{n-1} .
$$

The discriminant matrix of $f(x)$ is given by:

$$
\operatorname{Disc}(f)=\left(\begin{array}{ccccccc}
a_{0} & a_{1} & a_{2} & \cdots & a_{n} & & \\
0 & n a_{0} & (n-1) a_{1} & \cdots & a_{n-1} & & \\
0 & a_{0} & a_{1} & \cdots & a_{n-1} & a_{n} & \\
0 & 0 & n a_{0} & \cdots & 2 a_{n-2} & a_{n-1} & \\
& & & \cdots & & \cdots & \\
& & & \cdots & & \cdots & \\
& & & & a_{0} & a_{1} & \cdots a_{n} \\
& & & & 0 & n a_{0} & \cdots a_{n-1}
\end{array}\right) .
$$

For $k=1, \cdots, n$, let $\Delta_{k}(f)$ or simply, $\Delta_{k}$, denote the determinant of the submatrix of $\operatorname{Disc}(f)$ formed by the first $2 k$ rows and first $2 k$ columns. Note that $\Delta_{n}=\Delta=$ $\operatorname{det}(\operatorname{Disc}(f))$. The discriminant sequence of $f(x)$ is defined to be the sequence 
$\mathcal{S}=\left\{\Delta_{1}, \Delta_{2}, \cdots, \Delta_{n}\right\}$. The list $\left[\operatorname{sign}\left(\Delta_{1}\right), \cdots, \operatorname{sign}\left(\Delta_{n}\right)\right]$ is called the sign list of the discriminant sequence $\mathcal{S}$. Given a sign list $\left[s_{1}, s_{2}, \cdots, s_{n}\right]$, define the revised sign list as follows:

If $\left[s_{i}, s_{i+1}, \cdots, s_{i+j}\right]$ is a section of the given list, where

$$
s_{i} \neq 0, s_{i+1}=s_{i+2}=\cdots=s_{i+j-1}=0, s_{i+j} \neq 0,
$$

then we replace the subsection $\left[s_{i+1}, \cdots, s_{i+j-1}\right]$ by

$$
\left[-s_{i},-s_{i}, s_{i}, s_{i},-s_{i},-s_{i}, s_{i}, s_{i}, \cdots\right]
$$

i.e., let $e_{i+r}=(-1)^{\left[\frac{r+1}{2}\right]} s_{i}$ for $r=1,2, \cdots, j-1$. Otherwise let $e_{k}=s_{k}$. This gives us the revised sign list $\left[e_{1}, e_{2}, \cdots, e_{n}\right]$.

The following theorem is sufficient to determine the number of distinct real or imaginary roots, without considering multiplicities.

Theorem 2.1 (see [16, Theorem 1]). Given a polynomial $f(x)$ with real coefficients,

$$
f(x)=a_{0} x^{n}+a_{n-1} x^{n-1}+\cdots+a_{n},
$$

if the number of the sign changes of the revised sign list of

$$
\left\{\Delta_{1}(f), \Delta_{2}(f), \cdots, \Delta_{n}(f)\right\}
$$

is $p$, then the pairs of distinct conjugate imaginary roots of $f(x)$ equal $p$. Furthermore, if the number of non-vanishing members of the revised sign list is $q$, then the number of distinct real roots of $f(x)$ equals $q-2 p$.

Based on the above theorem and a longer round of computations, Hou-YangZeng defined the complete discrimination system, from which complete information about the numbers of real and imaginary roots of all possible multiplicities can be derived; cf. [16, p. 633]. Finally, we note the following result.

Theorem 2.2 (see [9, Number of Roots Theorem]). Let

$$
D_{n}=(-1)^{\frac{n(n-1)}{2}} a_{0}^{n-2} n^{-n} \Delta_{n} .
$$

Suppose the roots of $f(x)$ are distinct. Then the number of real roots of $f(x)$ is:

(1) if $n$ is odd, congruent to 1 or 3 modulo 4 according to whether $D_{n}>0$ or $D_{n}<0$;

(2) if $n$ is even, congruent to 0 or 2 modulo 4 according to whether $D_{n}$ and the leading coefficient of $f(x)$ have the same or opposite signs.

\subsection{Classification of isometries.}

Definition 2.3. A right eigenvalue $\lambda$ (counted without multiplicities) of $g \in$ $U(n, 1 ; \mathbb{F})$ is called negative, resp. positive, resp. null if the $\lambda$-eigenline is time-like, resp. space-like, resp. light-like.

Accordingly, a similarity class of eigenvalues $[\lambda]$ is called negative, positive, or null if its representative $\lambda$ is positive, negative, or null.

In the ball model of the hyperbolic space, by Brouwer's fixed point theorem, it follows that every isometry $g$ has a fixed point on the closure $\overline{\mathbf{H}_{\mathbb{F}}^{n}}=\mathbf{H}_{\mathbb{F}}^{n} \cup \partial \mathbf{H}_{\mathbb{F}}^{n}$. An isometry $g$ is called elliptic if it has a fixed point on $\mathbf{H}_{\mathbb{F}}^{n}$. It is called parabolic, resp. hyperbolic if it is non-elliptic, and has exactly one, resp. two fixed points on the boundary $\partial \mathbf{H}_{\mathbb{F}}^{n}$. From the conjugacy classification in $\mathrm{U}(n, 1 ; \mathbb{F})$, it follows that elliptic and hyperbolic elements are semisimple; cf. [8, Section-3]. Suppose $g$ is hyperbolic. Then $g$ has two similarity classes of null eigenvalues represented 
by $r e^{i \theta}, r^{-1} e^{i \theta}, r>1,0 \leq \theta \leq 2 \pi$ and, $(n-1)$ similarity classes of positive eigenvalues (which may not be different) of norm one. $g$ is regular hyperbolic if the null eigenvalues are represented by non-real complex numbers and the classes of positive eigenvalues are mutually disjoint. A non-regular hyperbolic has positive eigenvalue of multiplicity two. A non-regular hyperbolic isometry $g$ whose null eigenvalues are non-reals, is called semi-regular. $g$ is called screw hyperbolic if the null eigenvalues of $g$ are real numbers and it is called strictly hyperbolic if it is a screw hyperbolic and all positive eigenvalues are 1.

Let $g$ be elliptic. It follows from the conjugacy classification that $g$ has $n$ similarity classes of positive eigenvalues (which may not be different) and one similarity class of negative eigenvalues (which may coincide with one of the positive classes). All the eigenvalues of $g$ have norm 1 . An elliptic element is called regular if it has mutually disjoint classes of eigenvalues. An elliptic $g$ is called simple elliptic if it has only a single class of eigenvalues, i.e., it is of the form $\lambda I,|\lambda|=1$.

Suppose $g$ is parabolic. If $g$ is unipotent, i.e., all the eigenvalues are 1 , it is called a translation. A translation $g$ is called vertical, resp. non-vertical, if it has minimal polynomial $(x-1)^{2}$, resp. $(x-1)^{3}$. If $g$ is (non-unipotent) parabolic, then it has the Jordan decomposition $g=g_{s} g_{u}$, where $g_{s}$ is elliptic, $g_{u}$ is unipotent, and $g_{s}$ and $g_{u}$ commute in $\mathrm{U}(n, 1 ; \mathbb{F})$.

\section{Classification of QUaternionic hyperbolic isometries}

Let $A \in \operatorname{Sp}(n, 1)$. Write $\mathbb{H}=\mathbb{C} \oplus \mathbf{j} \mathbb{C}$. Express $A=A_{1}+\mathbf{j} A_{2}$, where $A_{1}, A_{2} \in$ $M_{2(n+1)}(\mathbb{C})$. This gives an embedding $A \mapsto A_{\mathbb{C}}$ of $\operatorname{Sp}(n, 1)$ into $\mathrm{GL}(2(n+1), \mathbb{C})$, cf. [18, section-2] and [26, section-2], where

$$
A_{\mathbb{C}}=\left(\begin{array}{cc}
A_{1} & -\overline{A_{2}} \\
A_{2} & \overline{A_{1}}
\end{array}\right) .
$$

We use the above embedding of $\operatorname{Sp}(n, 1)$ into $\operatorname{GL}(2(n+1), \mathbb{C})$. Note that the characteristic polynomial $\chi_{A_{\mathbb{C}}}(x)$ of $A_{\mathbb{C}}$ is an invariant of the conjugacy class of $A$. It follows from the conjugacy class representatives in $\operatorname{Sp}(n, 1)$ that $\chi_{A_{\mathbb{C}}}(x)$ is self-dual, i.e., if $\lambda \in \mathbb{C}$ is a root of $\chi_{A_{\mathbb{C}}}(x)$, so is $\lambda^{-1}$. Further if $\lambda$ is an eigenvalue, then so is $\bar{\lambda}^{-1}$; cf. [11, Lemma 6.2.5, p. 205]. It follows that if $\lambda$ is a root of the characteristic polynomial, so is $\bar{\lambda}$. Hence the characteristic polynomial of $A_{\mathbb{C}}$ is real and self-dual. Thus it has the form

$$
\chi_{A_{\mathbb{C}}}(x)=\sum_{j=0}^{2(n+1)} a_{j} x^{2(n+1)-j}, \text { where } a_{j}=a_{2(n+1)-j} ; a_{0}=a_{2(n+1)}=1,
$$

where for all $i, a_{i} \in \mathbb{R}$. Write $\chi_{A_{\mathbb{C}}}(x)=x^{n+1} g\left(x+x^{-1}\right)$, where

$$
g\left(x+x^{-1}\right)=\sum_{j=0}^{n} a_{j}\left(x^{n+1-j}+x^{-(n+1-j)}\right)+a_{n+1} .
$$

Expanding the terms in the brackets, and considering $t=x+x^{-1}$ as polynomial indeterminate, we get the polynomial

$$
g_{A}(t)=g\left(x+x^{-1}\right) .
$$

Using Newton's identities, the coefficients of $\chi_{A_{\mathbb{C}}}(x)$ can be expressed as a combination of several powers of $T_{k}=\operatorname{trace}\left(A_{\mathbb{C}}^{k}\right), k=1,2, \ldots, n+1$; cf. [19,21]. Hence the coefficients of $g_{A}(t)$ can be expressed by the numbers $T_{k}$. 
Theorem 3.1. Let $A$ be an element in $\operatorname{Sp}(n, 1)$. Suppose $A_{\mathbb{C}}$ is the corresponding element in $\operatorname{GL}(2(n+1), \mathbb{C})$. Let $\mathcal{S}_{A}=\left\{\Delta_{1}, \cdots, \Delta_{n+1}\right\}$ be the discriminant sequence of $g_{A}(t)$, where $\Delta_{n+1}=\Delta$ is the usual algebraic discriminant of $g_{A}(t)$. Let $D$ be the discriminant of the minimal polynomial of $A_{\mathbb{C}}$. Then the following holds.

(1) $A$ is regular hyperbolic if and only if $\Delta<0$.

(2) $A$ is regular elliptic if and only if $\Delta>0$.

(3) $A$ is semi-regular hyperbolic if and only if $\Delta=0$ and the number of sign changes of the revised sign list of $\mathcal{S}_{A}$ is exactly one.

(4) $A$ is screw hyperbolic if and only if $\Delta=0$ and $g_{A}(t)$ has a real root $\lambda$ such that $|\lambda|>2$.

(5) A is strictly hyperbolic if and only if $g_{A}(t)$ has a real root $\lambda$ such that $|\lambda|>2$ and for all $m \leq n-2, g_{A}^{(m)}(2)=0$.

(6) $A$ is elliptic or parabolic if and only if $\Delta=0$ and there is no sign change in the number of revised sign list of $\mathcal{S}_{A}$. Further, $A$ is parabolic if $D=0$; otherwise it is elliptic. Further, $A$ is simple elliptic if the number of nonvanishing members of the revised sign list is exactly one.

Proof. Since $\chi_{A_{\mathbb{C}}}(x)$ is a conjugacy invariant, so is $g_{A}(t)$. If $\alpha$ is a root of $\chi_{A_{\mathbb{C}}}(x)$, then $\alpha+\alpha^{-1}$ is a root of $g_{A}(t)$. Hence the nature of roots of $g_{A}(t)$ is determined by the nature of roots of $\chi_{A_{\mathbb{C}}}(x)$. It follows from the conjugacy classification in $\operatorname{Sp}(4,1)$ that for all $A$ in $\operatorname{Sp}(n, 1)$, the number of complex conjugate roots of $g_{A}(t)$ can be at most 2 .

For $A$ hyperbolic, the representatives of eigenvalues of $A$ are given by $r e^{i \theta}, r^{-1} e^{i \theta}$, $e^{i \phi_{k}}, k=1, \cdots, n-1$. It is easy to see from the embedding (3.1) that $\chi_{A_{\mathbb{C}}}$ has roots $r e^{ \pm i \theta}, r^{-1} e^{ \pm i \theta}, e^{ \pm i \phi_{k}}, k=1, \cdots, n-1$. Thus the roots of $g_{A}(t)$ are given by

$$
s_{1}=r e^{i \theta}+r^{-1} e^{-i \theta}, s_{2}=r^{-1} e^{i \theta}+r e^{-i \theta}, t_{k}=e^{i \phi_{k}}+e^{-i \phi_{k}}=2 \cos \phi_{k},
$$

for $k=1, \cdots, n-1$. Note that if $A$ is a screw hyperbolic, i.e., $\theta=0$ or $m \pi$, then $g_{A}(t)$ has at least one real double root $r+r^{-1}$ or $-r-r^{-1}$. Note that $\left|r+r^{-1}\right|>2$ and $\left|t_{k}\right| \leq 2$ for all $k$. Hence if $A$ is a screw hyperbolic, then $g_{A}(t)$ has exactly one double real root of absolute value $>2$. If $A$ is strictly hyperbolic, then 1 is a root of $A$ of multiplicity $n-1$. Hence 2 is a root of $g_{A}(t)$ of multiplicity $(n-1)$, and hence $g_{A}^{(m)}(2)=0$ for all $m \leq n-2$.

For $A$ elliptic or parabolic, the eigenvalues of $A$ are represented by $e^{i \theta_{i}}, i=$ $1, \cdots, n+1$, (for some $i, j, \theta_{i}$ may be equal to $\theta_{j}$ ). In this case, the roots of $g_{A}(t)$ are given by $u_{1}, \cdots, u_{n+1}$, where $u_{i}=2 \cos \theta_{i}$. For all $i,\left|u_{i}\right| \leq 2$.

If $A$ is regular hyperbolic, then $\theta \neq m \pi$. Hence $s_{1}$ and $s_{2}$ are non-real complex conjugate numbers and $g_{A}(t)$ has $n-1$ real roots. Depending on whether $n$ is even or odd, we have the following possibilities, and in each of the cases, applying Theorem 2.2 we see that $\Delta<0$ :

First note that $D_{n+1}=(-1)^{\frac{n(n+1)}{2}}(n+1)^{-(n+1)} \Delta_{n+1}$.

(i) For $n=4 k, n-1 \equiv 3 \bmod 4$ : Consequently, by Theorem 2.2, $D_{n+1}<0$. Since $\frac{4 k(4 k+1)}{2}=2 k(4 k+1)$ is an even number, hence $\Delta<0$.

(ii) For $n=4 k+1, n-1 \equiv 0 \bmod 4$ : Since the leading coefficient of $g_{A}(t)$ is $1>0$, hence by Theorem 2.2. $D_{n+1}>0$; consequently $\Delta<0$.

(iii) For $n=4 k+2, n-1 \equiv 1 \bmod 4$, hence $D_{n+1}>0$; consequently, $\Delta<0$.

(iv) For $n=4 k+3, n-1 \equiv 2 \bmod 4$, hence $D_{n+1}<0$; consequently, $\Delta<0$. 
TABLE 1. Classification of isometries of $\mathbf{H}_{\mathbb{H}}^{n}$.

\begin{tabular}{|c|c|}
\hline$\Delta$ & Type of isometry \\
\hline$<0$ & Regular Hyperbolic \\
\hline$>0$ & Regular elliptic \\
\hline$=0$ & Parabolic, non-regular elliptic or a non-regular hyperbolic \\
\hline
\end{tabular}

If $A$ is regular elliptic, then we have that $u_{i}$ 's are mutually distinct, hence all roots of $g_{A}(t)$ are real and mutually distinct. Using Theorem 2.2 and similar arguments as above, it follows that $\Delta>0$.

If $A$ is either of non-regular elliptic, non-regular hyperbolic or parabolic, then $g_{A}(t)$ has at least one root of multiplicity 2 . Hence $\Delta=0$.

Suppose $\Delta=0$. Then $\mathcal{S}_{A}=\left\{\Delta_{1}, \Delta_{2}, \cdots, \Delta_{n+1}\right\}$ be the discriminant sequence of the polynomial $g_{A}(t)$. Let $A$ be semi-regular hyperbolic. Then $g_{A}(t)$ has exactly two complex conjugate roots. Hence by Theorem 2.1 the number of sign changes of the revised sign list of $\mathcal{S}_{A}$ is exactly 1 . If $A$ is screw hyperbolic, elliptic or parabolic, then $g_{A}(t)$ has no complex roots, hence the number of sign changes of the revised sign list of $\mathcal{S}_{A}$ is zero. If $A$ is elliptic or parabolic, then from above we have seen that all the roots have absolute value $\leq 2$; A is screw hyperbolic if and only if $A$ has a root $\alpha$ such that $|\alpha|>2$.

Finally we note that if $A$ is parabolic, it has the Jordan decomposition $A=A_{s} A_{u}$, where $A_{u}$ is a vertical or non-vertical translation. Thus the minimal polynomial of $A_{\mathbb{C}}$ has a factor of the form $(x-\lambda)^{m}, m=2$ or 3 . Hence $D$ must be zero. For $A$ elliptic, the minimal polynomial of $A_{\mathbb{C}}$ is a product of distinct linear factors, hence $D \neq 0$. Suppose $A$ is simple elliptic. Then all the roots of $g_{A}(t)$ are equal, hence it has only one real root. Hence, by Theorem 2.1, the number of non-vanishing members of the revised sign list is exactly one.

This proves the theorem.

Note that Theorem 3.1 can also be adapted to the setting of complex hyperbolic geometry. This can be done by using the embedding of $\mathrm{U}(n, 1)$ into $\mathrm{GL}(2(n+1), \mathbb{R})$ given by (3.1) and then follow the same method as in the quaternionic case. In the action of $\mathrm{U}(n, 1)$ on $\mathbf{H}_{\mathbb{C}}^{n}$, the regular, semi-regular and (non-strictly) screw hyperbolic isometries fall in the same class; together we call them loxodromic. Also the simple elliptics, i.e., the scalar matrices of the form $\lambda I,|\lambda|=1$, acts as the identity on $\mathbf{H}_{\mathbb{C}}^{n}$. We have the following.

Corollary 3.2. Let $A$ be an element in $\mathrm{U}(n, 1)$. Suppose $A_{\mathbb{R}}$ is the corresponding element in $\operatorname{GL}\left((2(n+1), \mathbb{R})\right.$. Let $\mathcal{S}_{A}=\left\{\Delta_{1}, \cdots, \Delta_{n+1}\right\}$ be the discriminant sequence of $g_{A}(t)$, where $\Delta_{n+1}=\Delta$ is the usual algebraic discriminant of $g_{A}(t)$. Let $D$ be the discriminant of the minimal polynomial of $A_{\mathbb{R}}$. Then the following holds.

(1) $A$ is loxodromic if and only if one of the following holds:

(i) $\Delta<0$.

(ii) $\Delta=0$ and either the number of sign changes of the revised sign list of $\mathcal{S}_{A}$ is exactly one or, $g_{A}(t)$ has a real root $\lambda$ such that $|\lambda|>2$ and for some $m \leq n-2, g_{A}^{(m)}(2) \neq 0$.

(2) $A$ is regular elliptic if and only if $\Delta>0$. 
(3) $A$ is strictly hyperbolic if $g_{A}(t)$ has a real root $\lambda$ such that $|\lambda|>2$ and for all $m \leq n-2, g_{A}^{(m)}(2)=0$.

(4) $A$ is elliptic or parabolic if and only if $\Delta=0$ and there is no sign change in the number of revised sign list of $\mathcal{S}_{A}$. Further $A$ is parabolic if $D=0$; otherwise it is elliptic.

(5) $A$ acts as the identity if and only if $\Delta=0, D \neq 0$ and the number of non-vanishing members of the revised sign list is exactly one.

3.1. Remarks on a complete algorithm to classify the isometries. Theorem 3.1 gives us a fair classification of the isometries. However, it does not give us information about the multiplicities of the similarity classes of eigenvalues. However, following the methods in the above proof, using the polynomial $g_{A}(t)$ and the algorithm in [16, p. 633], it is indeed possible to derive a complete root classification of $g_{A}(t)$ with multiplicities. This will give us the number of distinct eigenvalues with multiplicities. For example, if $A$ is elliptic and $g_{A}(t)$ has distinct roots $2 \cos \theta_{1}, \cdots, 2 \cos \theta_{k}$ with multiplicities $m_{1}, \cdots, m_{k}$ respectively, then the eigenvalue classes of $A$ are represented by $e^{i \theta_{1}}, \cdots, e^{i \theta_{k}}$ each with multiplicities $m_{1}, \cdots, m_{k}$ respectively. Alternatively, the types of the isometries and the multiplicities of the similarity classes of eigenvalues can also be read out from the conjugacy classes of centralizers (or the $z$-classes) of the isometries. The conjugacy classes of centralizers in $\operatorname{Sp}(n, 1)$, resp. $\mathrm{U}(n, 1)$, have been classified in [14, resp. [4. Thus Theorem 3.1] the above algorithm, along with the $z$-classes, give us a complete algorithm to determine the type of an isometry of $\mathbf{H}_{\mathbb{H}}^{n}\left(\right.$ and also of $\mathbf{H}_{\mathbb{C}}^{n}$ ).

\section{REFERENCES}

[1] Lars V. Ahlfors, On the fixed points of Möbius transformations in $\mathbf{R}^{n}$, Ann. Acad. Sci. Fenn. Ser. A I Math. 10 (1985), 15-27. MR802464 (87c:20086)

[2] Daniel Allcock, New complex- and quaternion-hyperbolic reflection groups, Duke Math. J. 103 (2000), no. 2, 303-333, DOI 10.1215/S0012-7094-00-10326-2. MR:1760630 (2001f:11105)

[3] Alan F. Beardon, The geometry of discrete groups, Graduate Texts in Mathematics, vol. 91, Springer-Verlag, New York, 1983. MR698777 (85d:22026)

[4] Wensheng Cao and Krishnendu Gongopadhyay, Commuting isometries of the complex hyperbolic space, Proc. Amer. Math. Soc. 139 (2011), no. 9, 3317-3326, DOI 10.1090/S00029939-2011-10796-2. MR2811286 (2012h:51021)

[5] Wensheng Cao and Krishnendu Gongopadhyay, Algebraic characterization of isometries of the complex and the quaternionic hyperbolic planes, Geom. Dedicata 157 (2012), 23-39, DOI 10.1007/s10711-011-9599-7. MR2893478

[6] Wensheng Cao and John R. Parker, Jørgensen's inequality and collars in $n$-dimensional quaternionic hyperbolic space, Q. J. Math. 62 (2011), no. 3, 523-543, DOI 10.1093/qmath/haq003. MR2825470 (2012i:20065)

[7] Wensheng Cao, John R. Parker, and Xiantao Wang, On the classification of quaternionic Möbius transformations, Math. Proc. Cambridge Philos. Soc. 137 (2004), no. 2, 349-361, DOI 10.1017/S0305004104007868. MR2092064 (2005f:30082)

[8] S. S. Chen and L. Greenberg, Hyperbolic spaces, Contributions to analysis (a collection of papers dedicated to Lipman Bers), Academic Press, New York, 1974, pp. 49-87. MR.0377765 (51 \#13934)

[9] R. Dye and R. W. D. Nickalls, The geometry of the discriminant of a polynomial. The Mathematical Gazette, vol. 80, no. 488 (1996), 279-285.

[10] Georges Giraud, Sur certaines fonctions automorphes de deux variables, Ann. Sci. École Norm. Sup. (3) 38 (1921), 43-164 (French). MR1509233

[11] William M. Goldman, Complex hyperbolic geometry, Oxford Mathematical Monographs, The Clarendon Press Oxford University Press, New York, 1999. Oxford Science Publications. MR.1695450(2000g:32029) 
[12] Krishnendu Gongopadhyay, Algebraic characterization of the isometries of the hyperbolic 5space, Geom. Dedicata 144 (2010), 157-170, DOI 10.1007/s10711-009-9394-x. MR2580424 (2011a:57004)

[13] Krishnendu Gongopadhyay, Algebraic characterization of isometries of the complex and the quaternionic hyperbolic 3-spaces, Proc. Amer. Math. Soc. 141 (2013), no. 3, 1017-1027, DOI 10.1090/S0002-9939-2012-11422-4. MR3003693

[14] K. Gongopadhyay, The z-classes of quaternionic hyperbolic isometries, J. Group Theory, DOI 10.1515/jgt-2013-0013.

[15] K. Gongopadhyay, J. R. Parker and S. Parsad, On the classification of unitary matrices, Preprint.

[16] Lu Yang, Xiaorong Hou, and Zhenbing Zeng, A complete discrimination system for polynomials, Sci. China Ser. E 39 (1996), no. 6, 628-646. MR.1438754 (98b:68093)

[17] Inkang Kim and John R. Parker, Geometry of quaternionic hyperbolic manifolds, Math. Proc. Cambridge Philos. Soc. 135 (2003), no. 2, 291-320, DOI 10.1017/S030500410300687X. MR2006066 (2004h:53066)

[18] H. C. Lee, Eigenvalues and canonical forms of matrices with quaternion coefficients, Proc. Roy. Irish Acad. Sect. A. 52 (1949), 253-260. MR0036738 (12,153i)

[19] I. G. Macdonald, Symmetric functions and Hall polynomials, 2nd ed., Oxford Mathematical Monographs, The Clarendon Press Oxford University Press, New York, 1995. With contributions by A. Zelevinsky; Oxford Science Publications. MR.1354144 (96h:05207)

[20] G. D. Mostow, Strong rigidity of locally symmetric spaces, Princeton University Press, Princeton, N.J., 1973. Annals of Mathematics Studies, No. 78. MR0385004 (52 \#5874)

[21] Louis Halle Rowen, Polynomial identities in ring theory, Pure and Applied Mathematics, vol. 84, Academic Press Inc. [Harcourt Brace Jovanovich Publishers], New York, 1980. MR.576061 (82a:16021)

[22] S. Wolfram, "Mathematica: A System for Doing Mathematics by Computer," Wolfram Press, 2000.

[23] Masaaki Wada, Conjugacy invariants of Möbius transformations, Complex Variables Theory Appl. 15 (1990), no. 2, 125-133. MR1058518 (92a:30048)

[24] P. L. Waterman, Möbius transformations in several dimensions, Adv. Math. 101 (1993), no. 1, 87-113, DOI 10.1006/aima.1993.1043. MR1239454 (95h:30056)

[25] Wolfram Demonstration Project, The structure of real roots of a quintic polynomial, http://demonstrations. wolfram.com/TheStructureOfTheRealRootsOsAQuinticPolynomial/ Contributed by: Andrej Kozlowski.

[26] Fuzhen Zhang, Quaternions and matrices of quaternions, Linear Algebra Appl. 251 (1997), 21-57, DOI 10.1016/0024-3795(95)00543-9. MR1421264(97h:15020)

Indian Institute of Science Education and Research (IISER) Mohali, Knowledge

City, S.A.S. Nagar, Sector 81, P. O. Manauli, Pin 140306, India

E-mail address: krishnendug@gmail.com

Indian Institute of Science Education and Research (IISER) Mohali, Knowledge

City, S.A.S. Nagar, Sector 81, P. O. Manauli, Pin 140306, India

E-mail address: parsad.shiv@gmail.com 